\title{
Role of Arginase in Impairing Relaxation of Lung Parenchyma of Hyperoxia-Exposed Neonatal Rats
}

\author{
Nuzhat K.M. Ali ${ }^{\mathrm{a}}$ Anjum Jafri ${ }^{\mathrm{a}}$ Ramadan B. Sopi ${ }^{\mathrm{a}, \mathrm{c}}$ Y.S. Prakash ${ }^{\mathrm{b}}$ \\ Richard J. Martin ${ }^{\text {a }}$ Syed I.A. Zaidia \\ a Department of Pediatrics, Rainbow Babies and Children's Hospital, Case Western Reserve University, \\ Cleveland, Ohio, and ${ }^{\mathrm{b}}$ Departments of Anesthesiology, Physiology and Biomedical Engineering, Mayo Clinic

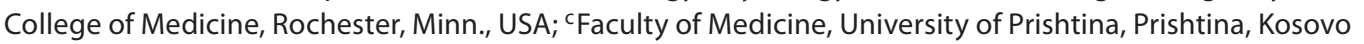

\section{Key Words}

Bronchodilation $\cdot$ Epithelium $\cdot$ L-Arginine $\cdot$ Nitric oxide production $\cdot \mathrm{N} \omega$-hydroxy-nor-arginine $\cdot$ Lung strips $\cdot$ Oxygen

\begin{abstract}
Background: Prolonged exposure of immature lungs to hyperoxia contributes to neonatal lung injury and airway hyperreactivity. We have previously demonstrated that neonatal exposure of rat pups to $\geq 95 \% \mathrm{O}_{2}$ impairs airway relaxation due to disruption of nitric oxide (NO)-cyclic guanosine monophosphate (cGMP) signaling. Objective: We now hypothesize that these impaired relaxation responses are secondary to hyperoxia-induced upregulation of arginase, which competes with NO synthase for L-arginine. Methods: Rat pups were exposed to moderate neonatal hyperoxia $\left(50 \% \mathrm{O}_{2}\right)$ or room air for 7 days from birth. In additional hyperoxic and room air groups, exogenous L-arginine $(300 \mathrm{mg} /$ $\mathrm{kg} /$ day i.p.) or arginase inhibitor ( $\mathrm{N} \omega$-hydroxy-nor-arginine, $30 \mathrm{mg} / \mathrm{kg} /$ day i.p.) were administered daily. After 7 days, animals were anesthetized and sacrificed either for preparation of lung parenchymal strips or lung perfusion. Results: In response to electrical field stimulation (EFS), bethanechol-pre-
\end{abstract}

constricted lung parenchymal strips from hyperoxic pups exhibited significantly reduced relaxation compared to room air controls. Supplementation of L-arginine or arginase blockade restored hyperoxia-induced impairment of relaxation. Expression of arginase I in airway epithelium was increased in response to hyperoxia but reduced by arginase blockade. Arginase activity was also significantly increased in hyperoxic lungs as compared to room air controls and reduced following arginase blockade. EFS-induced production of NO was decreased in hyperoxia-exposed airway smooth muscle and restored by arginase blockade. Conclusion: These data suggest that NO-cGMP signaling is disrupted in neonatal rat pups exposed to even moderate hyperoxia due to increased arginase activity and consequent decreased bioavailability of the substrate L-arginine. We speculate that supplementation of arginine and/or inhibition of arginase may be a useful therapeutic tool to prevent or treat neonatal lung injury.

Copyright $\odot 2011$ S. Karger AG, Basel

N.K.M.A., A.J. and R.B.S. contributed equally to this paper, and their names are presented in alphabetical order.

\section{KARGER}

두 2011 S. Karger AG, Basel

Fax +41613061234 E-Mail karger@karger.ch www.karger.com www.karger.com/neo
Syed I.A. Zaidi, PhD

Department of Pediatrics, Rainbow Babies and Children's Hospita

Case Western Reserve University, 11100 Euclid Avenue

Cleveland, OH 44106-6009 (USA)

Tel. +1 216844 7359, E-Mail syed.zaidi@case.edu 


\section{Introduction}

Bronchopulmonary dysplasia (BPD) is a substantial clinical problem in the preterm survivors of neonatal intensive care [1]. It is widely accepted that exposure to supplemental $\mathrm{O}_{2}$ contributes to neonatal lung injury. A consistent observation is that former preterm infants are at increased risk for reduced airway function and/or increased airway reactivity during childhood. The severity of this complication increases in preterm infants with a history of BPD [2, 3]. While BPD is likely multifactorial in origin, several studies, including our own, have focused on the effect of neonatal hyperoxia on airway structure and function [4-7]. Under both in vitro and in vivo conditions, increased constrictor responses of tracheal tissue and increased lung resistance have been demonstrated in rat pups exposed to $\geq 95 \% \mathrm{O}_{2}$ [4-6], consistent with the clinical course of survivors of BPD. BPD is associated with pathophysiologic changes in the lung parenchyma, likely implicating peripheral airway structures [1] and providing a rationale for the use of lung parenchymal strips to assess distal airway contractile and relaxant properties. Increased contractile responses are associated with decreased airway and lung tissue relaxant responses, suggesting that the balance of contraction versus relaxation of airway structures is shifted in response to $\geq 95 \% \mathrm{O}_{2}$ exposure [8-10]. The hyperoxia-induced contractile response was not observed when epithelium was removed from airways, suggesting a critical role of epithelium in hyperoxia-induced injury [10].

A major component of bronchodilation is nitric oxide (NO) derived from multiple sources including the epithelium and nonadrenergic noncholinergic innervations [11]. Impaired bronchodilation may reflect alterations in NO production or its subsequent effects on the airway. Inhalation of exogenous NO has been shown to restore distal lung growth in infant rats after neonatal hyperoxia [12]. In this regard, it is well established that the substrate for NO, L-arginine, can be metabolized by two major enzymes, NO synthase (NOS) and arginase, the latter of which is part of the urea cycle [13]. Thus, changes in the relative expression or activity of arginase versus NOS may alter the bioavailability of L-arginine for these enzymes and determine NO production. Hyperoxic exposure has been shown to increase arginase activity in the lung [14]. We have previously demonstrated that $\geq 95 \%$ $\mathrm{O}_{2}$ exposure of rat pups increases lung arginase activity; in turn, arginase competes with NOS for their common substrate L-arginine and decreases the bioavailability of $\mathrm{L}$-arginine. This may disrupt $\mathrm{NO}$-cyclic guanosine mo- nophosphate (cGMP) signaling and result in impaired airway relaxation [8]. These studies are consistent with a modulatory effect of NO on airway smooth muscle relaxation that is lost after hyperoxic exposure $[5,6,15]$. Prior data in a guinea pig model demonstrated that the expression of arginase was closely associated with airway NO depletion [16]. Decreased arginine bioavailability has been shown to be associated with increased arginase activity in asthma [17, 18], and blockade of arginase has been demonstrated to inhibit experimental asthma in animal models [19-21]. These studies encouraged us to explore the possibility that in vivo L-arginine supplementation or in vivo arginase blockade would protect neonatal lungs from hyperoxia-induced hyperreactivity.

In the clinical setting, prolonged exposure of neonates to moderate (e.g. $50 \%) \mathrm{O}_{2}$ is a common occurrence, in contrast to $\geq 95 \% \mathrm{O}_{2}$ exposure, which is uncommon for an extended period. Moderate elevation of inspired oxygen in rat pups induces a BPD-like lung morphology and hyperresponsiveness of tracheal rings and pulmonary arteries [4]. However, in these studies the relaxant properties of lung or airway structures were not investigated. Therefore, we sought to determine the effects of moderate hyperoxic exposure $\left(50 \% \mathrm{O}_{2}\right)$ on lung parenchymal relaxant responses, arginase expression and resultant NO production. We subsequently sought to restore the relaxant responses of lung parenchyma by supplementing rat pups with L-arginine in vivo and providing in vivo blockade of arginase to restore the relaxant responses of lung parenchyma.

\section{Materials and Methods}

Animals and Lung Parenchymal Strip Preparation

Experiments were performed in 7-day-old rat pups (SpragueDawley) employing protocols approved by the Institutional Animal Care and Use Committee of Case Western Reserve University, Cleveland, Ohio, USA. From the first day of life, the rat pups were exposed to hyperoxia $\left(50 \% \mathrm{O}_{2}\right)$ or room air for a period of 7 days. Rat pups from two different litters were randomly mixed and assigned to hyperoxic or room air groups on day 1 . Hyperoxic groups were housed with their mothers in a Plexiglas chamber (38 liters) and exposed to a continuous flow of $50 \% \mathrm{O}_{2}$ (2 liters/ $\min$ ) for 7 days. Mothers were rotated each day between room air and hyperoxic groups to protect them from a constant hyperoxic exposure. In additional groups of room air- or hyperoxia-exposed pups, subsets from each group were injected intraperitoneally each day with either normal saline, L-arginine $(300 \mathrm{mg} / \mathrm{kg})$ or $\mathrm{N} \omega$-hydroxy-nor-arginine (nor-NOHA; $30 \mathrm{mg} / \mathrm{kg}$ ) for arginase blockade. After 7 days, the animals were euthanized by asphyxiation using $\mathrm{CO}_{2}$. We chose a daily L-arginine dose of $300 \mathrm{mg} / \mathrm{kg}$ for 7 days in order to maintain an optimal level of substrate for 
NOS. This dose has been used previously by other investigators in different animal studies $[22,23]$ and no toxicity has been reported. Similarly, to maintain a steady plasma level of nor-NOHA, we used $30 \mathrm{mg} / \mathrm{kg} /$ day for 7 days to block arginase activity. This dose was selected based on other rodent studies [24,25].

\section{Physiological Studies}

Lung parenchymal strips of 1-mm thickness were obtained by sectioning the left lung in a parasagittal plane using a vibratome. Strips were anchored in a tissue bath and connected to a force displacement transducer (FT 03C; Grass Instruments Co.). The tissue bath was filled with Krebs-Henseleit solution maintained at $37^{\circ} \mathrm{C}$ and continuously oxygenated with a gas mixer $\left(95 \% \mathrm{O}_{2}\right.$ and $5 \%$ $\left.\mathrm{CO}_{2}\right)[8,9]$. An initial load of $0.2 \mathrm{~g}$ was used. The suspended lung parenchymal strips were rinsed every 20 min with Krebs-Henseleit solution and allowed to equilibrate for $60 \mathrm{~min}$ to establish a steady baseline. Lung parenchymal strip tension was recorded by Power Lab/4SP using Chart 5.0 Software (AD Instruments). A cumulative concentration-response curve was obtained to find an optimal concentration of bethanechol $\left(10^{-4} \mathrm{M}\right)$ that elicited $50-75 \%$ of the maximal contractile response in lung parenchymal strips. Electrical field stimulation (EFS) was applied through platinum wire electrodes to preconstricted lung parenchymal strips at various voltages $(5-50 \mathrm{~V} \mathrm{AC}$ at $60 \mathrm{~Hz})$ for $10 \mathrm{~s}$ at 2-min intervals to induce relaxation. The relaxation of strips in response to EFS was expressed as a percentage of the total preconstricted state for each strip as previously described by our group $[8,9]$.

\section{Immunostaining and Histology}

After 7 days of exposure to hyperoxia or room air, animals were anesthetized using a ketamine-xylazine cocktail and perfused through the right ventricle with phosphate-buffered saline (PBS; pH 7.4) containing $10 \mathrm{U} / \mathrm{ml}$ heparin followed by $4 \%$ paraformaldehyde in PBS ( $\mathrm{pH}$ 7.4). The airways of lungs were inflated with $4 \%$ paraformaldehyde at $12 \mathrm{~cm} \mathrm{H}_{2} \mathrm{O}$, tied and removed for 24 -hour postfixation. Lungs were then cryoprotected in 30\% sucrose in PBS, and $10-\mu \mathrm{m}$ coronal sections were cut. The sections were washed in PBS and incubated for $30 \mathrm{~min}$ in PBS containing $0.3 \%$ Triton X-100. To block nonspecific binding sites, the sections were incubated for $1 \mathrm{~h}$ with 5\% normal goat serum for arginase I and $\alpha$-smooth muscle actin and 5\% normal donkey serum for arginase II. After a further wash with PBS, the sections were incubated overnight at $4^{\circ} \mathrm{C}$ in mouse anti-arginase I (1:200; BD Transduction Laboratory), mouse anti- $\alpha$-smooth muscle actin (Sigma Chemical Co.) or rabbit anti-arginase II antibodies (Santa Cruz Biotechnology). The sections were washed in PBS-Triton X-100 and incubated with secondary antibody (1:200; goat anti-mouseFITC for arginase I and $\alpha$-smooth muscle actin or donkey antirabbit-RITC for arginase II). Airways were identified by immunostaining with anti-tubulin IV antibody, which detects ciliated airway epithelial cells. Airway smooth muscle was stained with $\alpha$-smooth muscle actin. Sections were mounted using antifade mounting medium and examined under a fluorescent microscope. Hematoxylin and eosin staining and $\alpha$-smooth muscle actin immunostaining were performed on 4 - $\mu \mathrm{m}$-thick adjacent paraffin sections.

\section{Arginase Assay}

Lungs of rat pups were removed and snap frozen in liquid nitrogen. Arginase activity in lung tissue was measured by the method of Jenkinson and Grigor [26] as described in our previous publications $[8,9]$. In brief, an arginase assay was performed in lung homogenates in the presence of L-arginine, and urea as the end product was measured using a urea standard curve. Baseline urea concentration in each sample was also measured in samples without L-arginine. The protein content of tissue samples was measured, and arginase activity was expressed as nanomoles of urea per minute per milligram of protein.

\section{In situ Measurement of NO Production in Airway}

Smooth Muscle

NO production in airway smooth muscle was measured using the NO-sensitive fluorescent dye 4,5-diaminofluorescein diacetate (DAF-2DA; Invitrogen) as described previously [27]. Briefly, lung strips $(100 \mu \mathrm{m})$ were incubated with $10 \mu \mathrm{M}$ DAF-2DA at $37^{\circ} \mathrm{C}$ for $45 \mathrm{~min}$ and then thoroughly rinsed. Using a Nikon Diaphot microscope $(\times 40 / 1.3$ numerical aperture oil immersion lens, 12-bit cooled CCD camera, MetaFluor Software; Universal Imaging, Downington, Pa., USA), airways within DAF-2DA-loaded strips were identified by bright-field imaging and the smooth muscle layer was delineated. The DAF-2DA was then visualized (488 nm excitation, $515 \mathrm{~nm}$ emission) within the airway smooth muscle as an index of baseline NO levels. Graded incremental EFS $(2-50 \mathrm{~V})$ was applied to the lung strips (as described above). Following the final EFS protocol, DAF-2DA fluorescence intensity was re-evaluated by epifluorescence microscopy. The net NO produced due to EFS was estimated as the difference from baseline (arbitrary units) and is presented as the mean $\pm \operatorname{SEM}(\mathrm{n}=3)$. In a recent study [27], we calibrated DAF-2DA fluorescence to estimate actual NO levels and found that amplitudes similar to those reported here correspond to $10-50 \mathrm{nM} N O$, which is within the physiological range.

\section{Statistical Analysis}

The results are expressed as means \pm SEM. Statistical significance was determined by two-way ANOVA with repeated measurements to determine the effect of EFS on relaxation responses between hyperoxic versus room air groups. To analyze differences between individual voltages, post hoc comparison using the Tukey-Kramer multiple comparison test was performed. For arginase activity, one-way ANOVA followed by post hoc comparison using the Tukey test was performed. $\mathrm{p}<0.05$ was considered statistically significant.

\section{Results}

\section{Effect of in vivo L-Arginine Supplementation on \\ Relaxation of Lung Parenchymal Strips}

As shown in figure 1, greater relaxation was observed in lung parenchymal strips from room air $(n=18)$ versus hyperoxic $(\mathrm{n}=12)$ pups $(\mathrm{p}<0.01)$. There was a significantly greater impairment of relaxation with EFS of 45 and $50 \mathrm{~V}$ (both $\mathrm{p}<0.01$ ) in hyperoxic versus room air rat pups. The changes at 5-40 V were not significantly different. In vivo L-arginine supplementation to hyperoxic pups $(n=12)$ restored the impaired relaxation of 


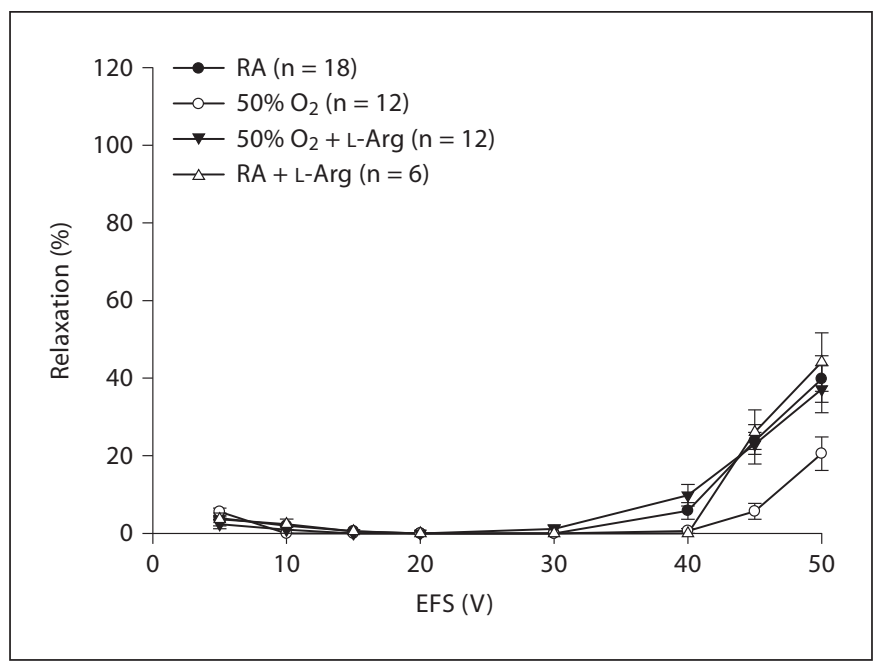

Fig. 1. Effect of in vivo L-arginine supplementation on relaxation of lung parenchymal strips. Impaired relaxation of lung parenchymal strips in response to EFS was observed in rat pups exposed to moderate hyperoxia for 7 days when compared to room air controls $(\mathrm{p}<0.01)$. There was significantly impaired relaxation with EFS of 45 and $50 \mathrm{~V}$ (both $\mathrm{p}<0.01$ ) in hyperoxic versus room air rat pups. In vivo supplementation of L-arginine $(300 \mathrm{mg} / \mathrm{kg} /$ day $)$ for 7 days restored the impairment of relaxation in hyperoxic pups to the room air level. RA = Room air.

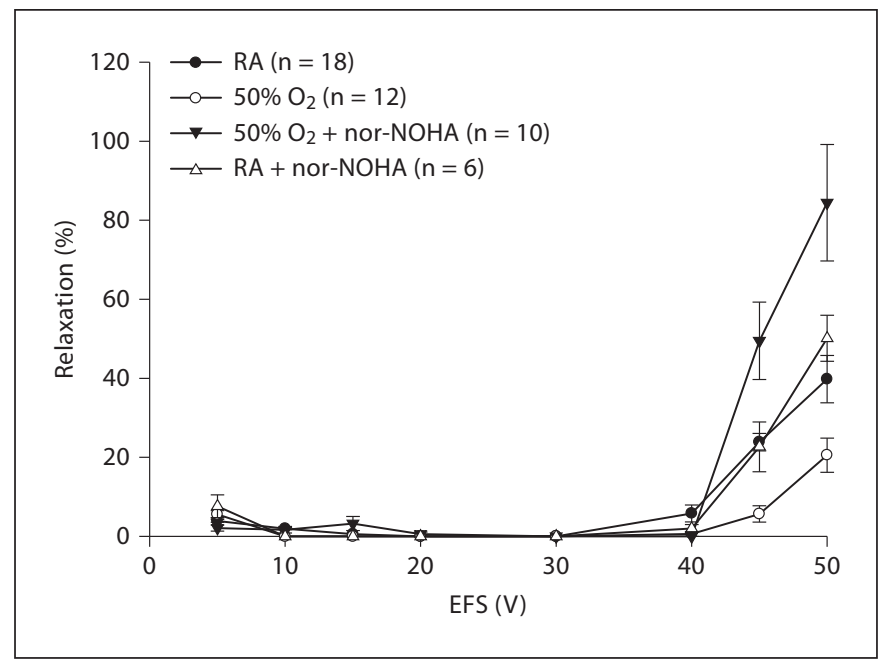

Fig. 2. Effect of in vivo blockade of arginase on relaxation of lung parenchyma. In vivo administration of the arginase inhibitor norNOHA (30 mg/kg/day for 7 days) not only restored the impaired relaxation of hyperoxia-exposed lung strips but enhanced relaxation above room air control values (at 45 and $50 \mathrm{~V}$; $\mathrm{p}<0.01$ ). The administration of nor-NOHA did not have any effect on room air animals. $\mathrm{RA}=$ Room air.
Fig. 3. Effect of supplementation of L-arginine and nor-NOHA on arginase I expression in the lung (immunostaining). The arginase I isoform was expressed in the airway epithelial layer of room air-exposed animals. Hyperoxic exposure increased the expression of arginase I in the airway epithelial layer. L-Arginine supplementation of hyperoxia-exposed pups restored arginase I expression to that of room air controls. The administration of nor-NOHA to hyperoxic animals reduced arginase expression below that of room air controls. Bar $=75 \mu \mathrm{m}$.
Room air

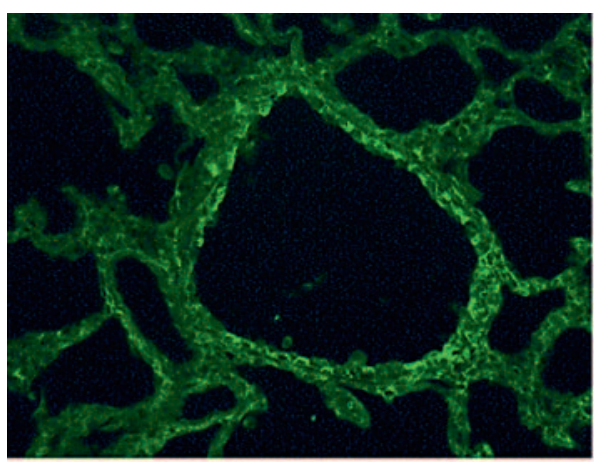

$50 \% \mathrm{O}_{2}+$ L-arginine

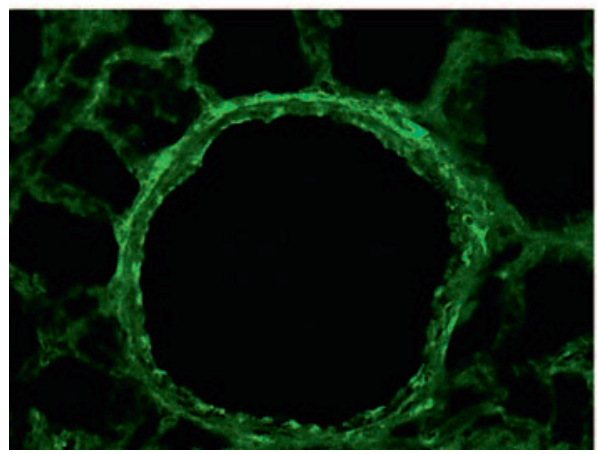

Hyperoxia $\left(50 \% \mathrm{O}_{2}\right)$

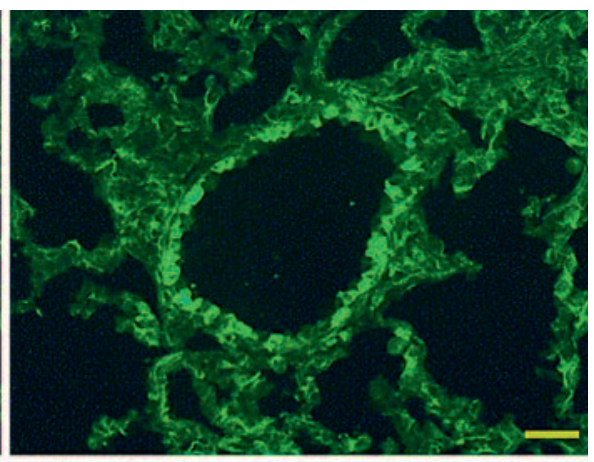

$50 \% \mathrm{O}_{2}+$ nor- $\mathrm{NOHA}$

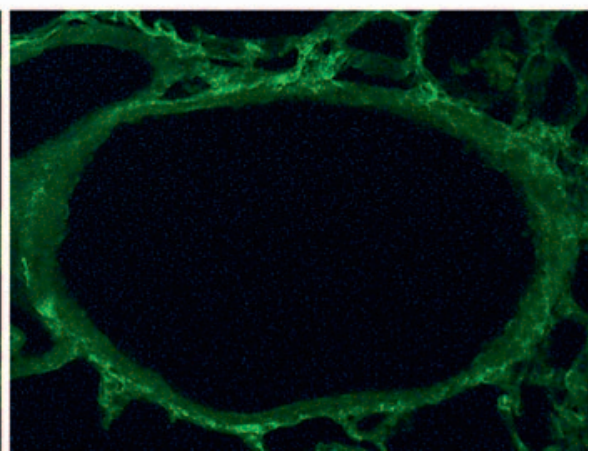


lung strips to their room air level (fig. 1). This effect of $\mathrm{L}$-arginine was significant at 45 and $50 \mathrm{~V}$ (both $\mathrm{p}<0.01$ ) when arginine-supplemented hyperoxic pups were compared to pups exposed to hyperoxia alone. In room air animals, L-arginine supplementation in vivo $(n=6)$ did not change the relaxation responses to EFS in preconstricted strips from room air-exposed controls. D-Arginine, a metabolically inactive isoform of L-arginine, also did not induce any significant changes when given to room air pups (data not shown).

\section{Effect of in vivo Blockade of Arginase on Relaxation of} Lung Parenchymal Strips

Arginase blockade using nor-NOHA was used to determine whether this may enhance the availability of $\mathrm{L}$ arginine for NOS thus resulting in improved EFS-induced relaxation responses of hyperoxia-exposed lungs. As noted above, hyperoxia impaired relaxation in preconstricted strips when compared to room air controls. EFS-induced relaxation was significantly increased in hyperoxia-exposed pups after arginase blockade when compared to pups exposed to hyperoxia alone $(n=10$; $\mathrm{p}<0.01)$. The nor-NOHA administration in vivo not only restored the impaired relaxation of hyperoxia-exposed lung strips to the room air level but actually enhanced relaxation above that of room air controls, which was significant at 45 and $50 \mathrm{~V}$ (both $\mathrm{p}<0.01$; fig. 2). When norNOHA was administered to room air animals $(n=6)$, it had no significant effect on the EFS-induced relaxation of lung strips (fig. 2).

\section{Effect of Supplementation of L-Arginine or}

Administration of nor-NOHA on Lung Arginase

Expression and Histology

The arginase I isoform was found to be expressed in the airway epithelial layer of room air-exposed animals via immunostaining. Moderate hyperoxic exposure increased the expression of arginase I in the epithelial lining of airways. Representative data from 1 of 5 pups are shown in figure 3. L-Arginine supplementation of hyperoxia-exposed pups restored the expression of arginase I in airway epithelial cells to the room air level. Interestingly, the expression of epithelial arginase I was reduced below the room air level in nor-NOHA-treated hyperoxia-exposed animals. The arginase II isoform was not expressed in airways but showed generalized expression in parenchymal tissue including the endothelial lining of blood vessels (fig. 4). Hyperoxic exposure also increased the expression of arginase II in blood vessels, which was restored to the room air level by the in vivo supplementa- tion of L-arginine or by in vivo blockade of arginase by nor-NOHA. Moderate hyperoxia did not produce any measurable structural changes in airways and lung parenchyma. This is demonstrated in figure 5, which is representative of the histologic data collected in 5 pups. Furthermore, supplementation of L-arginine or administration of nor-NOHA to hyperoxic pups also did not produce any observable changes in airway and lung architecture (fig. 5).

\section{Effect of Supplementation of L-Arginine and nor-NOHA on Arginase Activity of Lung}

We sought to determine whether increased arginase expression in airway epithelial cells was associated with increased arginase activity in whole lung of hyperoxiaexposed pups. Arginase activity was measured in wholelung homogenates ( $n=5$ per group). Moderate hyperoxic exposure caused an overall increase in arginase activity in hyperoxic lungs as compared to room air-exposed lungs ( $\mathrm{p}<0.05$; fig. 6). Supplementation of L-arginine to hyperoxic animals did not significantly change arginase activity when compared to hyperoxia alone. Administration of nor-NOHA to hyperoxic animals decreased lung arginase activity; however, the values remained higher than room air levels.

\section{Effect of Supplementation of L-Arginine and nor-NOHA on NO Production in the Airway \\ Smooth Muscle Layer}

NO production in response to EFS ( $\mathrm{n}=3$ per group) was reduced in the airway smooth muscle layer of lung strips obtained from hyperoxic animals $(p<0.05)$. Supplementation of L-arginine and arginase blockade had no effect on NO production in airway smooth muscle of room air-exposed animals. However, in hyperoxic animals L-arginine supplementation partially restored $\mathrm{NO}$ production, and arginase blockade completely restored NO production in airway smooth muscle ( $\mathrm{p}<0.05$; fig. 7 ).

\section{Discussion}

BPD is a substantial clinical problem in the preterm survivors of neonatal intensive care [1], and increased airway reactivity is a well-recognized complication in this high-risk population [3]. The pathophysiology of BPD comprises a combination of factors including inflammatory injury, mechanical ventilation and a high concentration of inspired oxygen superimposed on an immature lung. Commonly, $\geq 95 \% \mathrm{O}_{2}$ exposure has been used in 
Fig. 4. Effect of supplementation of L-arginine and nor-NOHA on arginase II expression in the lung (immunostaining). The arginase II isoform was not expressed in the airway epithelial layer of room airor hyperoxia-exposed animals. It was expressed in the endothelial lining of blood vessels (arrows), and its expression was increased by hyperoxic exposure. Both L-arginine supplementation and administration of nor-NOHA to hyperoxia-exposed pups reduced arginase II expression. $\beta$-Tubulin IV is expressed in ciliated cells of airway epithelium and was used to identify airway structures. $\mathrm{A}=$ Airway; $\mathrm{B}=$ blood vessel. $\mathrm{Bar}=75 \mu \mathrm{m}$.

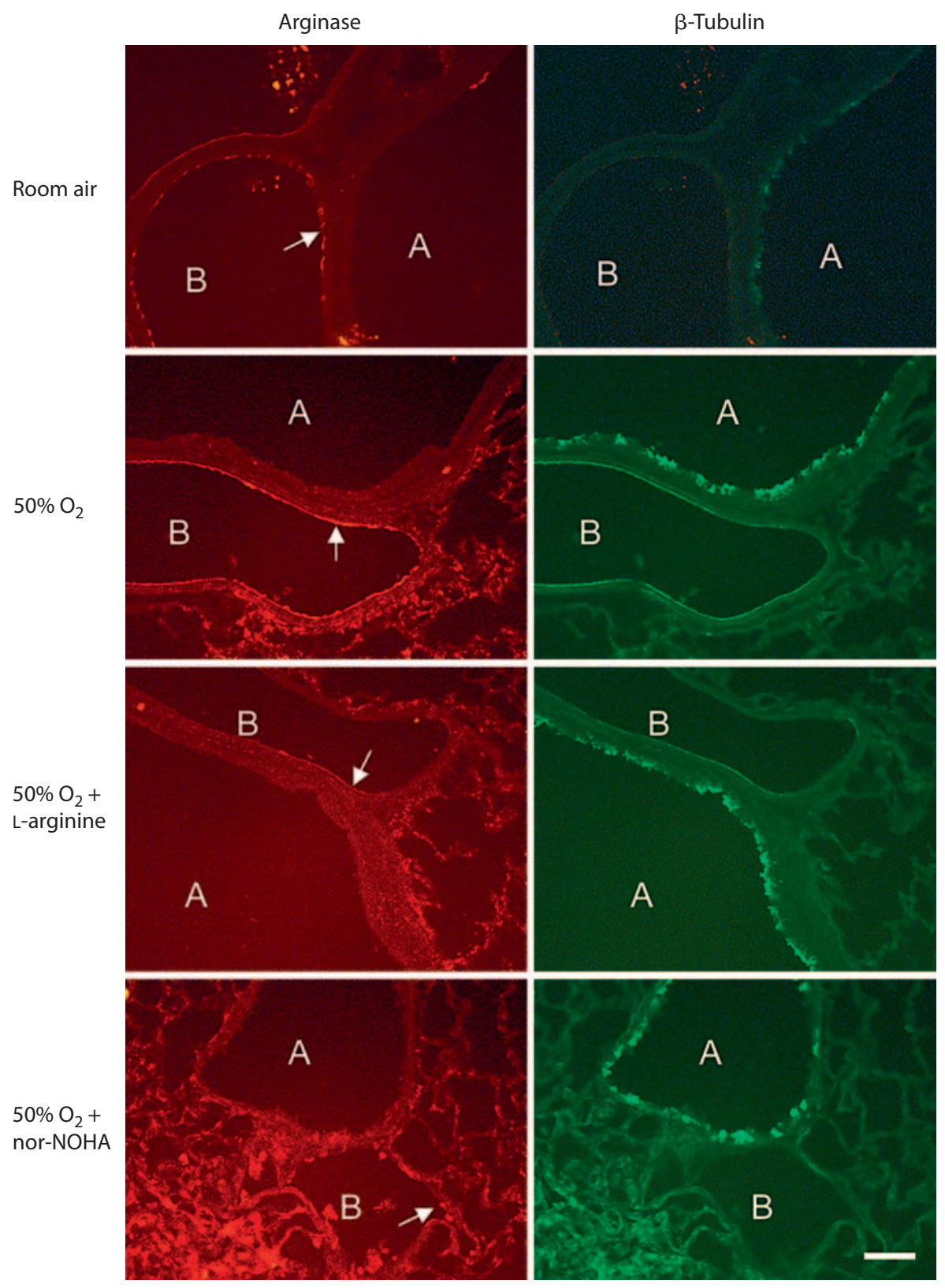

experimental animals to produce a BPD-like developmental model [8-10]. However, in the clinical setting, sustained exposure frequently involves a lower concentration of oxygen (e.g. 40-60\%), which has also been shown to induce hyperresponsiveness and airway inflammation in a rat model [28]. Accordingly, in this study, we used a more clinically relevant concentration of $50 \%$ $\mathrm{O}_{2}$. We hypothesized that $50 \% \mathrm{O}_{2}$ exposure to immature lungs would impair airway smooth muscle relaxant re- sponses, contributing to the increased airway reactivity that is a consequence of neonatal lung injury.

Our results demonstrate that 7-day exposure of newborn rat pups to $50 \%$ oxygen did not produce any measurable structural changes in airways or in lung parenchyma; however, it did cause a significant impairment of EFS-induced relaxation of preconstricted lung strips. These data are consistent with our previous studies using $\geq 95 \% \mathrm{O}_{2}$; in those studies, we demonstrated that expo- 


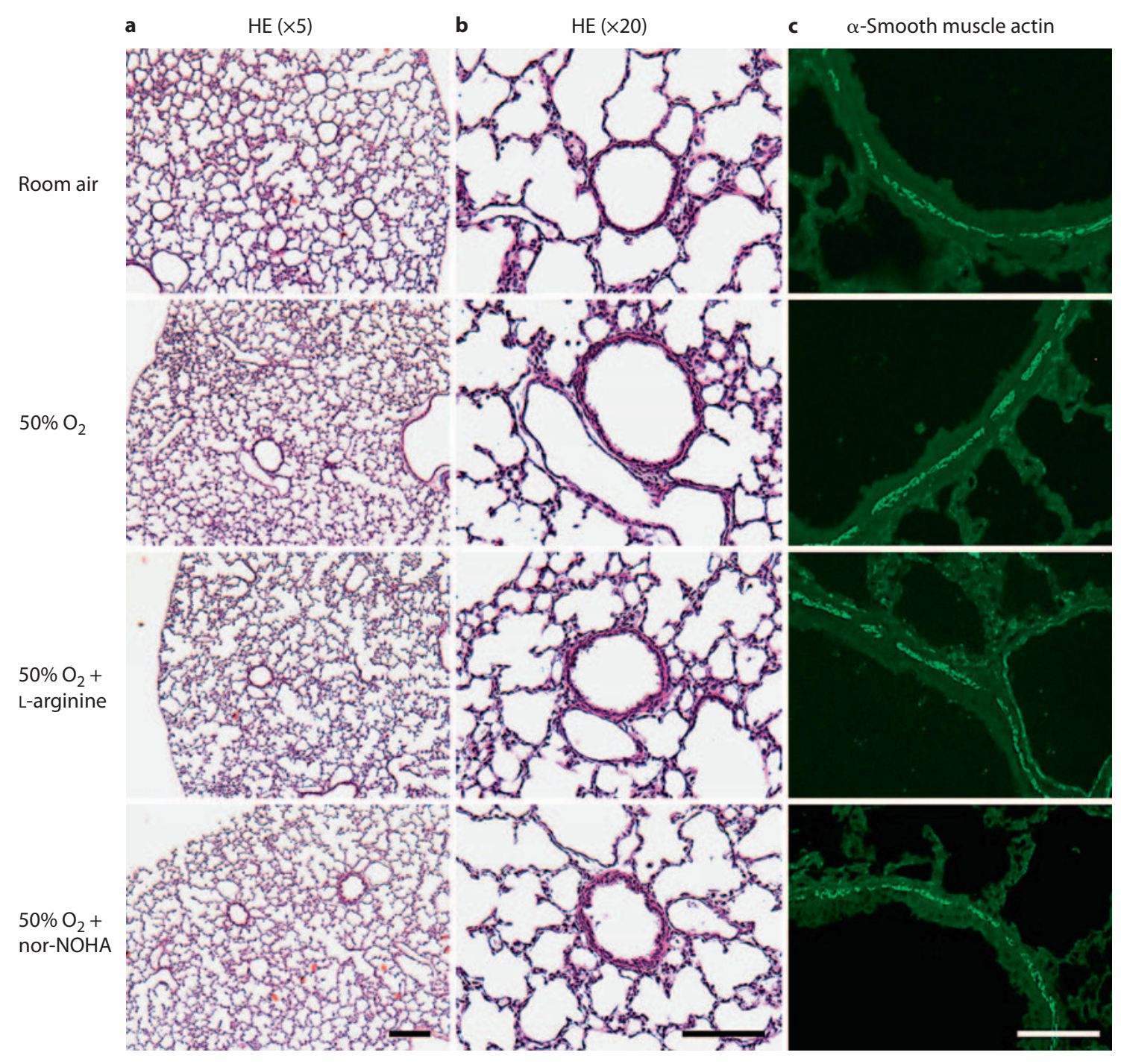

Fig. 5. Effect of moderate hyperoxia $\left(50 \% \mathrm{O}_{2}\right)$ on lung histology. a Hematoxylin and eosin (HE) staining of $4-\mu \mathrm{m}$-thick paraffin-embedded sections showing no observable changes in lung parenchyma and airways between groups. b Higher magnification of a showing airways. There were no observable histological changes in airway structures. c Immunostaining of adjacent sections with $\alpha$-smooth muscle actin antibody showing no changes in the thickness or architecture of the airway smooth muscle layer. Bar $=200 \mu \mathrm{m}(\mathbf{a}, \mathbf{b})$ and $100 \mu \mathrm{m}(\mathbf{c})$.

sure to hyperoxia impairs relaxation of tracheal smooth muscle [5] as well as lung parenchymal strips $[8,9]$, potentially via impairment of NO-cGMP signaling. In one of our previous studies [8], we also demonstrated that neonatal exposure to $\geq 95 \% \mathrm{O}_{2}$ in rat pups increases arginase activity, which, in turn, may decrease the bioavailability of L-arginine for NO-cGMP signaling, resulting in airway hyperreactivity and impaired relaxation of lung parenchymal tissue. As NO is produced from L-arginine via NOS [13], the bioavailability of L-arginine, a common substrate for both NOS and arginase, may play a key role in the production of NO. We therefore sought to determine whether in vivo intraperitoneal administration of exogenous L-arginine to hyperoxic animals can restore NO production and hyperoxia-induced impairment of airway smooth muscle relaxation to room air levels by increasing the bioavailability of L-arginine for NO-cGMP signaling. In order to maintain an optimal level of sub- 


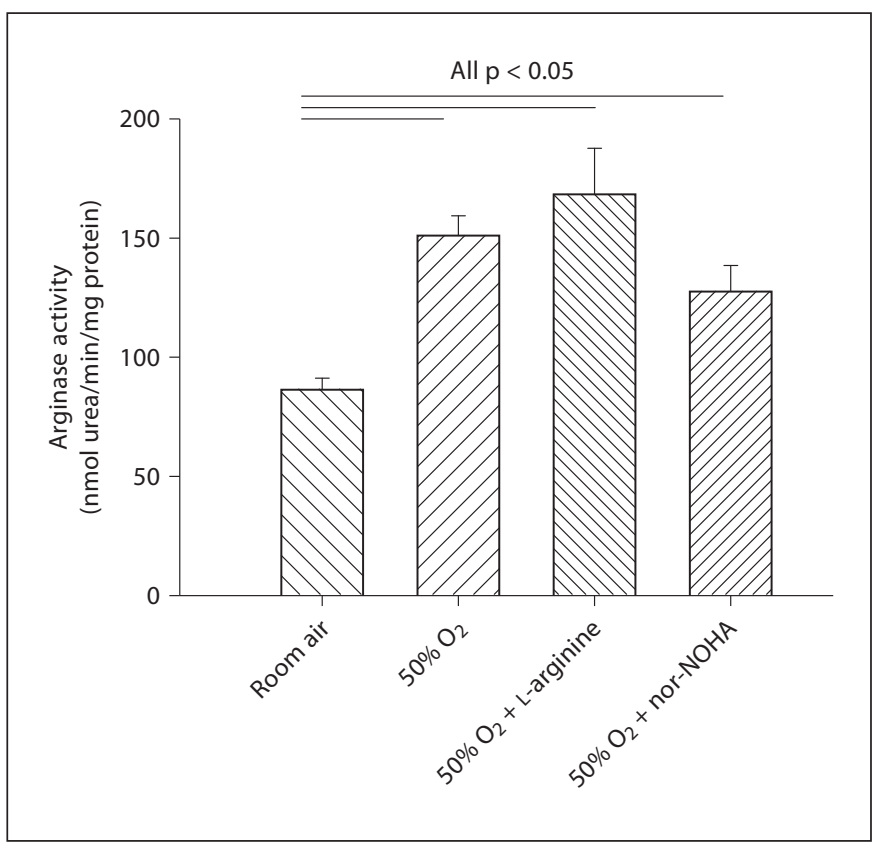

Fig. 6. Effect of moderate hyperoxia $\left(50 \% \mathrm{O}_{2}\right)$ on arginase activity of the lung. In hyperoxic lungs, arginase activity was significantly increased $(\mathrm{p}<0.05)$ as compared to room air-exposed lungs $(\mathrm{n}=5$ per group). Supplementation of L-arginine in hyperoxic animals $(n=5)$ did not significantly change arginase activity when compared to hyperoxia alone, and it remained significantly higher compared to room air controls $(\mathrm{p}<0.05)$. The administration of nor-NOHA to hyperoxic animals reduced the arginase activity; however, the values remained higher than room air levels. Data expressed as means $\pm \mathrm{SE}$.

strate for NOS, we injected $300 \mathrm{mg} / \mathrm{kg}$ L-arginine daily for 7 days. This dose did not exhibit any apparent toxicity in other studies $[22,23]$. We also did not observe any adverse affects of L-arginine supplementation on feeding or other behavior or any other obvious symptoms of stress in these rat pups. Consistent with our hypothesis, this protocol of daily in vivo supplementation of L-arginine was able to partially restore both NO production and lung strip relaxation in pups exposed to moderate hyperoxia.

We sought to determine whether in vivo arginase inhibition may serve as an alternative approach to restore airway relaxation via NO-cGMP signaling in hyperoxia-exposed pups. We used $30 \mathrm{mg} / \mathrm{kg} /$ day nor-NOHA to block arginase activity based on other rodent studies [24, $25]$. We chose to utilize nor-NOHA because it has a low median inhibition concentration (low clearance rate from the lung) and it does not directly interact as either

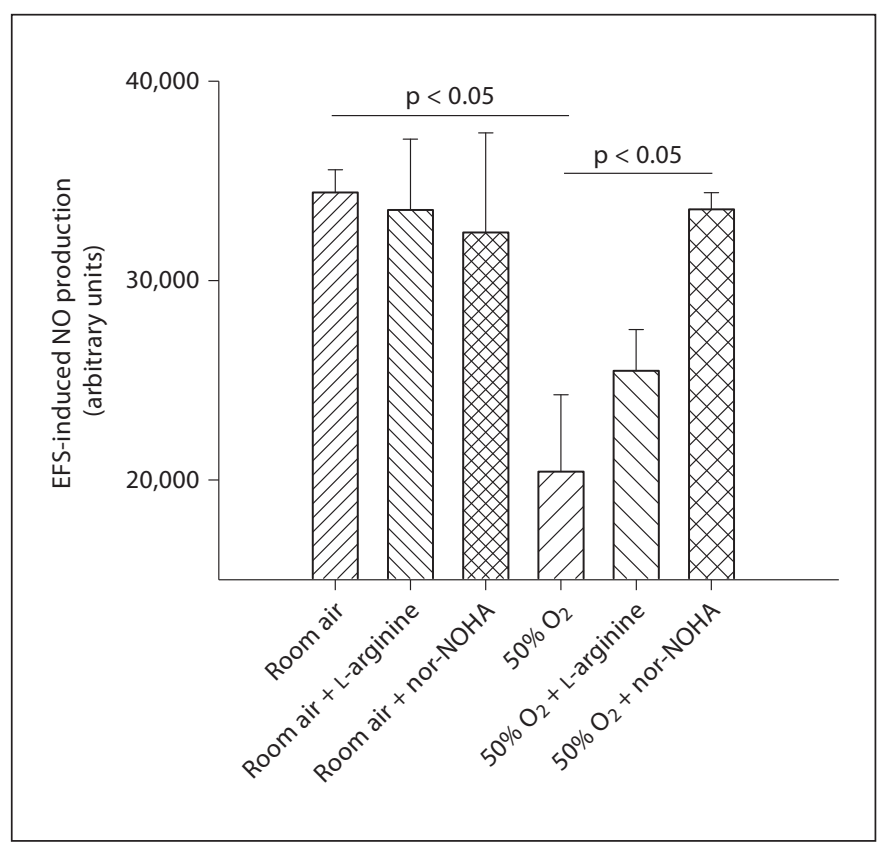

Fig. 7. Effect of moderate hyperoxia (50\% oxygen) on EFS-induced NO production in airway smooth muscle. EFS-induced NO production was decreased in airway smooth muscle of lung strips from hyperoxic pups as compared to lung strips from room airexposed pups ( $\mathrm{n}=3$ per group; $\mathrm{p}<0.05$ ). Supplementation of $\mathrm{L}-$ arginine and arginase blockade had no effect on NO production in room air-exposed animals. In hyperoxic animals, L-arginine supplementation appeared to partially restore NO production in airway smooth muscle. Arginase blockade completely restored NO production in airway smooth muscle $(n=3 ; p<0.05)$. Data expressed as means $\pm \mathrm{SE}$

a substrate or an inhibitor of NOS activity when compared to NOHA [29]. Thus, the possibility of increased NO production due to mechanisms other than arginase inhibition is unlikely in our study. Consistent with our physiologic data, inhibition of arginase activity by norNOHA in hyperoxic animals restored NO production in airway smooth muscle. Interestingly, nor-NOHA not only reversed the impaired relaxation but it relaxed the hyperoxia-exposed parenchymal strips more than the room air control strips. This observation and immunostaining data suggest that nor-NOHA decreased arginase activity in airway compartments of hyperoxia-exposed animals below baseline. In contrast to our immunostaining and physiologic data, NO production was restored and did not exceed room air levels after arginase inhibition in hyperoxia-exposed pups. Therefore, an alternative mechanism such as downstream cGMP signaling might be implicated in this enhanced response to arginase inhi- 
bition. Another possibility is that in room air-exposed animals, L-arginine may be present in abundance and thus may not be a limiting substrate for NOS in the production of NO. However, in hyperoxic animals, due to increased arginase activity the L-arginine is reduced and probably became a limiting substrate. Therefore, any increase in L-arginine content due to inhibition of arginase activity may have a profound effect on NO production after hyperoxic exposure.

Arginase I was expressed in airway epithelial cells and was increased in the hyperoxic lungs. Expression of arginase I in the hyperoxic group was restored to room air levels by L-arginine supplementation, which correlated with our physiologic data. We propose that this normalization of relaxation of lung parenchyma is due to increased production of NO by supplemental L-arginine in the hyperoxic animals. This is consistent with our results that show that NO production was reduced in airway smooth muscle of hyperoxia-exposed lungs and restored by nor-NOHA administration. The observed enhancement of relaxation in hyperoxia-exposed lung parenchyma in response to EFS after administration of nor-NOHA is supported by immunostaining data, according to which nor-NOHA reduced the expression of arginase I in alveolar epithelium below baseline room air levels. Arginase II may not play a direct role in airway relaxation in our model due to its absence in airway epithelium.

We have also demonstrated that moderate hyperoxic exposure increases arginase activity. Lung arginase activity remained elevated in L-arginine-supplemented hyperoxic animals, which was contrary to the physiologic data and also contrary to the restoration of arginase expression to room air levels as observed in epithelial cells. This suggests that the total arginase activity of lung homogenate may include circulating plasma arginase isoforms and cannot be correlated with the relaxant responses of lung parenchyma. Reduction in the expression of arginase I after arginine supplementation could reflect the metabolism of $\mathrm{L}$-arginine toward NOS production in a compartmentalized pool of airway epithelial cells which is not reflected in lung tissue as a whole [17]. We cannot exclude other mechanisms that might decrease NO production due to uncoupling of NOS or decreased levels of tetrahydrobiopterin as a result of its increased reactive oxygen-dependent consumption with a resultant reduction in NOS activity [30]. We do recognize that lung parenchymal strips contain various anatomic constituents including alveolar, bronchial and blood vessel walls as well as connective and interstitial cells with varying contractile properties. However, rodent parenchymal strips provide a useful model to measure actual tissue mechanical properties, and our prior experiments $[8,9]$ demonstrate that this preparation can be utilized under wellcontrolled conditions in the absence of circulating elements and centrally mediated neuronal components. Future studies might employ isolated peripheral airways, although this is a challenge given the small size of rat pups.

Our animal model has proven quite successful to characterize the role of modest hyperoxic exposure in enhancing airway reactivity via downregulation of a functional NO-mediated signaling pathway. We recognize that this model cannot precisely simulate the multifactorial contributors to BPD [1]. Our observation that the lung parenchymal structures in this model appeared histologically relatively intact may also be a factor that differentiates it from BPD, although human histologic data from subjects with milder degrees of BPD are largely unavailable. More importantly, our rat pup model provides a potential mechanism for characterizing the increased airway reactivity observed in preterm infants with neonatal lung injury.

In conclusion, 7-day exposure of newborn rat pups to moderate hyperoxia impairs the relaxant responses of lung parenchyma and increases arginase I expression in airway epithelial cells. The resultant increase in arginase activity results in a reduction in the bioavailability of Larginine for NOS, which, in turn, results in impaired NO production and impaired NO-cGMP signaling. We have recently documented that inhalation of exogenous $\mathrm{NO}$ in preterm infants at risk for BPD decreases the need for bronchodilator therapy at 1 year of age [31]. Supplementation of L-arginine or blockade of arginase may provide alternative approaches to enhance endogenous NO production and restore impaired relaxant responses in preterm infants at risk for increased airway reactivity.

\section{Acknowledgements}

This work was supported by NIH grant HL-56470 and an Investigator-Initiated Grant from IKARIA (ZAIDIS1). The authors acknowledge Mrs. Mary Ann O'Riordan and Juliann Di Fiore for statistical analysis, Lucas W. Meuchel, Michael A. Thompson and Philippe Delmotte for NO measurements and Shi Jian Liu for technical assistance. 


\section{References}

$\checkmark 1$ Jobe AH, Bancalari E: Bronchopulmonary dysplasia. Am J Respir Crit Care Med 2001; 163:1723-1729.

-2 Hack M, Taylor HG, Drotar D, Schluchter M, Cartar L, Andreias L, Wilson-Costello D, Klein N: Chronic conditions, functional limitations and special health care needs of school-aged children born with extremely low-birth-weight in the 1990s. JAMA 2005; 294:318-325.

\3 Pelkonen AS, Hakulinen AL, Turpeinen M: Bronchial lability and responsiveness in school children born very preterm. Am J Respir Crit Care Med 1997;156:1178-1184.

$\checkmark 4$ Belik J, Jankov RP, Pan J, Tanswell AK: Chronic $\mathrm{O}_{2}$ exposure enhances vascular and airway smooth muscle contraction in the newborn but not adult rat. J Appl Physiol 2003;94:2303-2312.

$\checkmark 5$ Mhanna MJ, Haxhiu MA, Jaber MA, Walenga RW, Chang C-H, Liu S, Martin RJ: Hyperoxia impairs airway relaxation in immature rats via a cAMP-mediated mechanism. J Appl Physiol 2004;96:1854-1860.

-6 Potter CF, Kuo N-T, Farver CF, McMahon JT, Chang C-H, Agani FH, Haxhiu MA, Martin $\mathrm{RJ}$ : Effects of hyperoxia on nitric oxide synthase expression, nitric oxide activity, and lung injury in rat pups. Pediatr Res 1999;45: $8-13$.

7 Warner BB, Stuart LA, Papes RA, Wispé JR: Functional and pathological effects of prolonged hyperoxia in neonatal mice. Am J Physiol 1998;275:L110-L117.

$\checkmark 8$ Sopi RB, Haxhiu MA, Martin RJ, Dreshaj IA, Kamath S, Zaidi SI: Disruption of NO-cGMP signaling by neonatal hyperoxia impairs relaxation of lung parenchyma. Am J Physiol Lung Cell Mol Physiol 2007;293:L1029L1036.

$\checkmark 9$ Sopi RB, Martin RJ, Haxhiu MA, Dreshaj IA, Yao Q, Jafri A, Zaidi SI: Role of brain-derived neurotrophic factor in hyperoxia-induced enhancement of contractility and impairment of relaxation in lung parenchyma. Am J Physiol Lung Cell Mol Physiol 2008; 295:L348-L355.

$\checkmark 10$ Solway J, Hershenson MB: Structural and functional abnormalities of the airways of hyperoxia-exposed immature rats. Chest 1995;107(3 suppl):89S-93S.
1 Belvisi MG, Stretton D, Barnes PJ: Nitric oxide as an endogenous modulator of cholinergic neurotransmission in guinea-pig airways. Eur J Pharmacol 1991;198:219-221.

12 Lin YJ, Markham NE, Balasubramanium V, Tang JR, Maxey A, Kinsella JP, Abman SH: Inhaled nitric oxide enhances distal lung growth after exposure to hyperoxia in neonatal rats. Pediatr Res 2005;58:22-29.

13 Murad F: Shattuck Lecture. Nitric oxide and cyclic GMP in cell signaling and drug development. N Engl J Med 2006;355:2003-2011.

14 Que LG, Kantrow SP, Jenkinson CP, Piatadosi CA, Huang Y-C: Induction of arginase isoforms in the lung during hyperoxia. Am J Physiol 1998;275:L96-L102.

15 Iben SC, Dreshaj IA, Farver CF, Haxhiu MA, Martin RJ: Role of endogenous nitric oxide in hyperoxia induced airway hyperreactivity in maturing rats. J Appl Physiol 2000;89: 1205-1212.

16 Meurs H, McKay S, Maarsingh H, Hamer MA, Macic L, Molendijk N, Zaagsma J: Increased arginase activity underlies allergeninduced deficiency of cNOS-derived nitric oxide and airway hyperresponsiveness. Br J Pharmacol 2002;136:391-398.

17 Morris SM Jr: Recent advances in arginine metabolism: roles and regulation of the arginases. Br J Pharmacol 2009;157:922-930.

18 Morris CR, Poljakovic M, Lavrisha L, Machado L, Kuypers FA, Morris SM Jr: Decreased arginine bioavailability and increased serum arginase activity in asthma. Am J Respir Crit Care Med 2004;170:148-153.

19 Maarsingh H, Zuidhof AB, Bos IS, van Duin M, Boucher JL, Zaagsma J, Meurs H: Arginase inhibition protects against allergen-induced airway obstruction, hyperresponsiveness, and inflammation. Am J Respir Crit Care Med 2008;178:565-573.

20 North ML, Khanna N, Marsden PA, Grasemann H, Scott JA: Functionally important role for arginase I in airway hyperresponsiveness of asthma. Am J Physiol Lung Cell Mol Physiol 2009;296:L911-L920.

-21 Takahashi N, Ogino K, Takemoto K, Hamanishi S, Wang D, Takigawa T, Shibamor M, Ishiyama H, Fujikura Y: Direct inhibition of arginase attenuated airway allergic reactions and inflammation in a Dermatophagoides farinae-induced NC/Nga mouse model. Am J Physiol Lung Cell Mol Physiol 2010; 299:L17-L24.
22 Sheridan BC, McIntyrre RC, Meldrum DR, Fullerton DA: L-Arginine prevents lung neutrophil accumulation and preserves pulmonary endothelial function after endotoxin. Am J Physiol 1998;274:L337-L342.

23 Strapkova A, Antosova M, Nosalova G: Relation of L-arginine to airway hyperreactivity. Gen Physiol Biophys 2008;27:85-91.

24 Bratt JM, Franzi LM, Linderholm AL, O'Roark EM, Kenyon NJ, Last JA: Arginase inhibition in airways from normal and nitric oxide synthase 2-knockout mice exposed to ovalbumin. Toxicol Appl Pharmacol 2010; 242:1-8.

$>25$ Bagnost T, Berthelot A, Bouhaddi M, Laurant $\mathrm{P}$, Andre C, Guillaume Y, Demougeot C: Treatment with arginase inhibitor $\mathrm{N}$ [omega]hydroxy-nor-L-arginine improves vascular function and lowers blood pressure in adult spontaneously hypertensive rat. J Hypertens 2008;26:1110-1118.

$>26$ Jenkinson CP, Grigor MR: Rat mammary arginase isolation and characterization. Biochem Med Metab Biol 1994,51:156-165.

27 Meuchel LW, Thompson MA, Pabelick CM, Prakash YS: Neurotrophins induce nitric oxide in pulmonary endothelial cells. Cardiovasc Res, in press.

-28 Denis D, Fayon MJ, Berger P, Molimard M, DeLara MT, Roux E, Marthan R: Prolonged moderate hyperoxia induces hyperresponsiveness and airway inflammation in newborn rats. Pediatr Res 2001;50:515-519.

29 Moali C, Boucher JL, Sari MA, Stuehr DJ, Mansuy D: Substrate specificity of NO synthases: detailed comparison of L-arginine, homo-L-arginine, their $\mathrm{N}$ omega-hydroxy derivatives, and N omega-hydroxynor-L-arginine. Biochemistry 1998;37:10453-10460.

30 Bevers LM, Braam B, Post JA, vanZonneveld AJ, Rabelink TJ, Koomans HA, Verhaar MC, Joles JA: Tetrahydrobiopterin, but not L-arginine, decreases NO synthase uncoupling in cells expressing high levels of endothelial NO synthase. Hypertension 2006;47:87-94.

-31 Hibbs AM, Walsh MC, Martin RJ, Truog WE, Lorch SA, Alessandrini E, Cnaan A, Palermo L, Wadlinger SR, Coburn CE, Ballard PL, Ballard RA: One-year respiratory outcomes of preterm infants enrolled in the Nitric Oxide (to prevent) Chronic Lung Disease trial. J Pediatr 2008;153:525-529. 